\title{
Study on Communication Methods for Electric Power High-voltage Equipment Monitoring System
}

\author{
https://doi.org/10.3991/ijoe.v14i02.8212 \\ Yuchen Jia, Jia Tao, Limin Huo $\left.{ }^{(}\right)$ \\ Agricultural University of Hebei, Baoding, China \\ jiayuchen $1981 @ 163 . \mathrm{com}$
}

\begin{abstract}
Real-time monitoring of high-voltage equipment in substations is beneficial for early detection of faults. The use of wireless sensor networks to build monitoring system is an effective way, but the data collection is a difficult task. The author introduces a real-time monitoring system based on ZIGBEE and mobile communication technology. The system includes multiple monitoring points and terminal platforms. Each monitoring point consists of a number of sensor nodes to form a ZIGBEE network, detecting relevant parameters, coordinator node data collected one by one, known as linear transmission, and finally to the monitoring platform through the mobile communication network. This paper presents a fusion algorithm for monitoring cell data acquisition to reduce the amount of data uploaded to the base station. In addition, multi-hop routing algorithm based on opportunistic routing is proposed to balance network energy and improve network transmission rate and efficiency.
\end{abstract}

Keywords-Electric Power High-voltage Equipment; ZIGBEE; Fuzzy Comprehensive; Extremely Opportunistic Routing

\section{Introduction}

High-voltage equipment, such as transformers, switchgear, circuit breakers, is an important part of the power system. Its smooth operation is an important guarantee for the normal supply of electricity. Monitoring and overhauling high-voltage equipment is an important part of routine maintenance. With the development of the Internet of Things, monitoring of high-voltage equipment using sensors and networks has become the focus of research. The advantage is that it saves manpower and material resources, and the monitoring data are objective and effective. There have been many related technologies produced. Design and develop a remote system for continuous monitoring of leakage currents and ground currents in high voltage electrical substations. Based on wireless local area network technology, the system can be used to monitor continuously a variety of plants within the substation and has low power consumption with inbuilt overvoltage protection ${ }^{[1]}$. A method for powering an on-line condition-monitoring device installed on a high-voltage transmission tower is presented. The technique uses the harvesting plate and a transformer both installed away from the transmission line to harvest the electric field energy ${ }^{[2]}$. 
In the application of wireless sensor networks, there have been some problems. Due to the collection points are too much and large amount of data are collected, realtime data upload will bring the network congestion. In addition, if each monitoring unit communicates with the server directly, the workload of the server is too large, it will inevitably lead to network congestion; if upgrade server configuration, it will make the system cost-effective decline.This paper presents a fusion algorithm for monitoring cell data collection to reduce the amount of data uploaded to the base station. In addition, a multi-hop routing algorithm based on opportunistic routing is proposed to balance network energy and improve network transmission rate and efficiency. Monitoring system is shown in Figure 1.

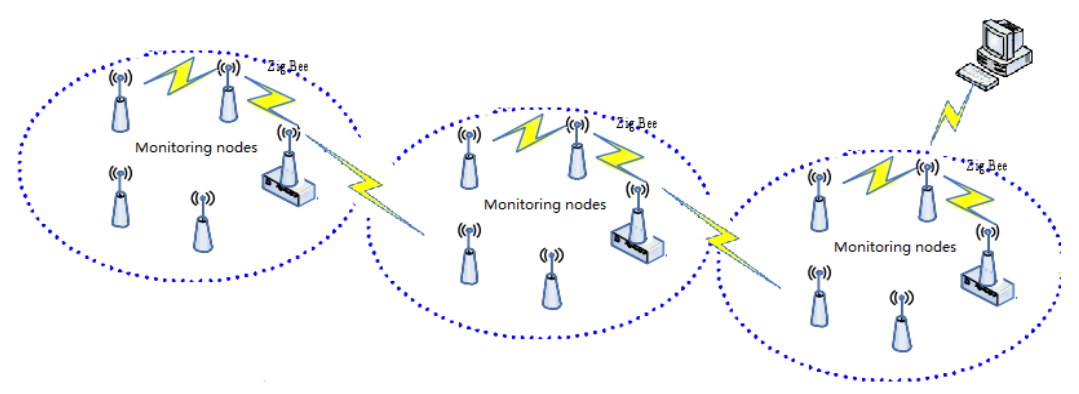

Fig. 1. Monitoring system based on ZIGBEE

\section{Design of acquisition node communication}

ZIGBEE is a low-cost, low-power, wireless mesh network standard targeted at the wide development of long battery life devices in wireless control and monitoring applications. The ZIGBEE network layer natively supports both star and tree networks, and generic mesh networking ${ }^{[3]}$. Every network must have one coordinator device, tasked with its creation, the control of its parameters and basic maintenance [4]

\subsection{Design of Endnotes}

The end node's processor and information delivery device uses the CC2530EB, a truly IEEE-enabled 802.15.4 system that supports proprietary IEEE 802.15.4 and ZIGBEE, ZIGBEE PRO and ZIGBEE RF4CE standards ${ }^{[5]}$, using on-chip RF to send data to the coordinator, operating frequency of $2.4 \mathrm{GHz}$.

In order to save energy, in the software design, the end node set two types of threshold. The first is the data threshold. When more than 10 sets of the same data are collected, the node enters the sleep state for 60 minutes. The second is the data transmission threshold. When the collected data is within a certain range, it stops to send data to the coordinator. Such as the collection of temperature, when it is between $25^{\circ}$ and $30^{\circ}$, the data sending is stopped. The data processing flow after the end node joins the network is shown in Figure 2. 


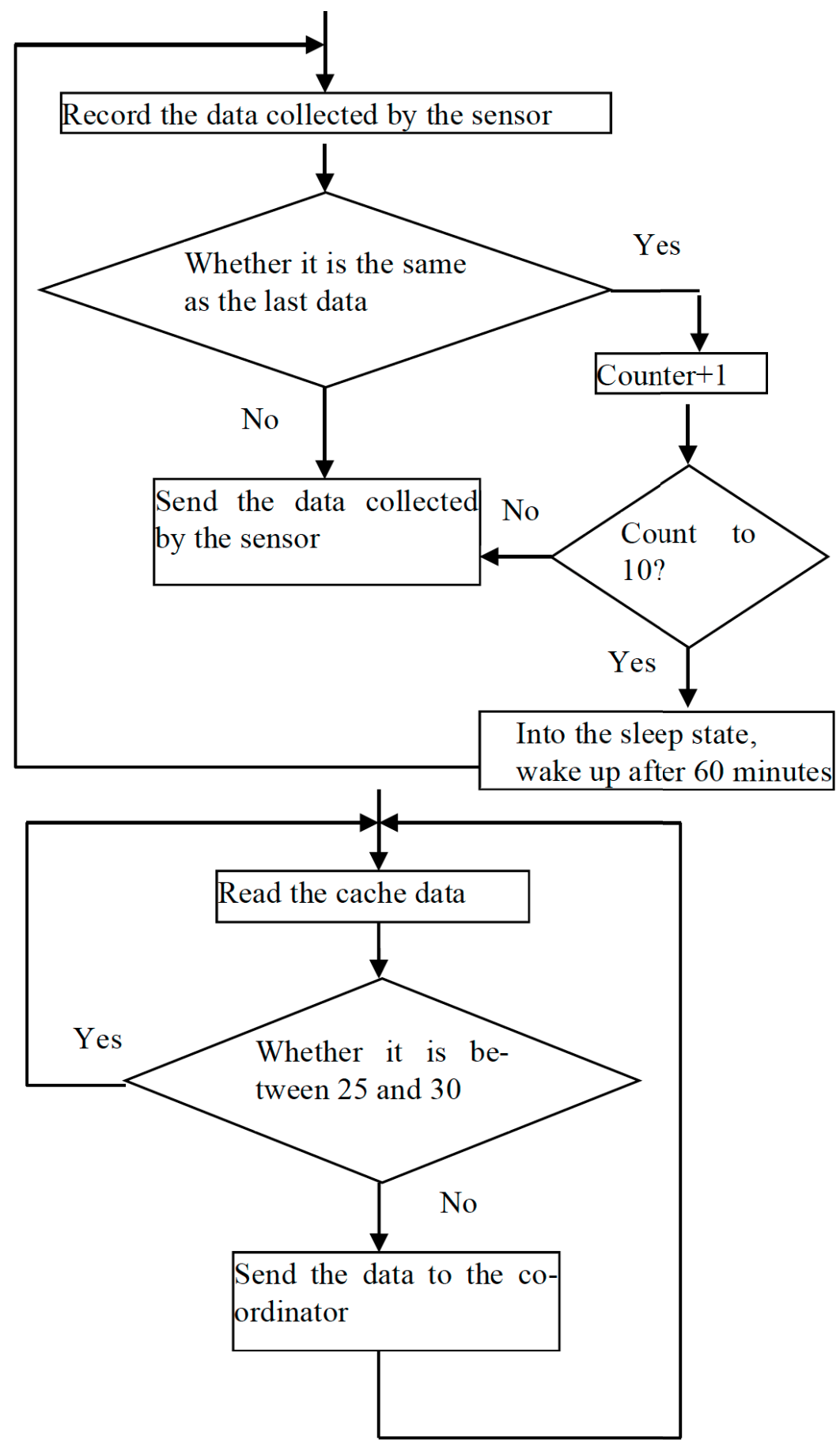

Fig. 2. Signal processing flow in the end node 


\subsection{Design of Coordinator}

The coordinator is responsible for identifying the terminal node and assigning the channel and network PAN ID to the end node. It is the core of the star topology network, hardware of that is CC2530, equipped with TI Z-Stack 2.3.1, and set to FFD (Full Function Device). The coordinator receives data from sixteen nodes in the experiment which is divided into four categories, each of which collects the same type of data, and it requires the first level of data fusion to obtain the effective value of such parameters; for different types of data, it needs to be carried out the second level of data fusion, in order to obtain the comprehensive assessment of the water quality of the region. Therefore, in addition to building ZIGBEE network, the coordinator also needs to integrate the data of the collected data, in order to reduce the amount of data and to ensure the rate of data transmission and real-time ${ }^{[6]}$. Taking into account the ZIGBEE node energy consumption constraints, the first level of data fusion uses the algorithm of statistical average, and the second level of data fusion uses the algorithm of adaptive weighting and fuzzy comprehensive evaluation.

$$
\begin{gathered}
\bar{x}=\frac{1}{n} \sum_{i=1}^{n} x_{i} \\
s=\left[\frac{1}{n-1} \sum_{i=1}^{n}\left(x_{i}-\bar{x}\right)^{2}\right]^{\frac{1}{2}}
\end{gathered}
$$

Where, $\mathrm{n}$ is the number of sensors and $\mathrm{x}$ is the data matrix.

The fuzzy comprehensive evaluation model was established by adding the weight coefficient to the four types of monitoring data as the evaluation index. The evaluation results (excellent, good, medium and poor) were uploaded. If the evaluation is excellent, the coordinator sleeps for 30 minutes; if the evaluation is poor, the sensor data is uploaded. The process of fuzzy comprehensive evaluation is:

1) Standardization of evaluation index data.

$$
R=\left[\begin{array}{l}
R_{1} \\
R_{2} \\
R_{3} \\
R_{4}
\end{array}\right]=\left[\begin{array}{llll}
x_{11} & x_{12} & x_{13} & x_{14} \\
x_{21} & x_{22} & x_{23} & x_{24} \\
x_{31} & x_{32} & x_{33} & x_{34} \\
x_{41} & x_{42} & x_{43} & x_{44}
\end{array}\right]
$$

2) Determine the weighting function. The statistical average and standard deviation of the first level are introduced into the weighting function as their dynamic parameters. The dynamic weighting function is the partial normal distribution function and it is as (4). 


$$
w_{i}(x)=\left\{\begin{array}{lll}
0, & \text { if } & x \leq \partial_{i} \\
1-e^{-\left(\frac{x-\partial_{i}}{\sigma_{i}}\right)^{2}}, & \text { if } & x>\partial_{i}
\end{array}\right.
$$

3) After experimental analysis, it identifies $M(\bullet, \oplus)$ as fuzzy operator.

4) In accordance with the principle of maximum membership to make a comprehensive evaluation, the results of the judge $S$ is:

$$
S=W \circ R=\left[\begin{array}{llll}
w_{1} & w_{2} & w_{3} & w_{4}
\end{array}\right] \circ\left[\begin{array}{l}
R_{1} \\
R_{2} \\
R_{3} \\
R_{4}
\end{array}\right]=\left[\begin{array}{llll}
s_{1} & s_{2} & s_{3} & s_{4}
\end{array}\right]
$$

The method of fuzzy comprehensive evaluation is used to fuse the data, which obviously reduces the amount of data transmission and has a significant effect on extending the life cycle of the network.

\section{Construction of Multi-hop Routing Protocol}

According to the design goal, this system is used in substations with high voltage equipment. The multiple monitoring units deployed are unevenly distributed and the environment is complicated. The data from monitoring unit uploading and the commands release from the monitoring platform require a stable and reliable communication network. The ZIGBEE data acquisition unit set up above is battery powered and the energy is limited. At the same time, the coordinator node takes into account the work of data fusion, and the energy consumption in data communication is more limited. Therefore, in the formation of communication network, in addition to guarantee the rate, it needs to consider the issue of energy consumption. In the construction of communication network, it uses stratified multi-hop routing protocol according to the distance, and it designs an opportunistic routing algorithm based on the shortest path and the residual energy of the node. The coordinator in the monitoring unit becomes the routing node, and the sink in the network is composed of the LTE module.

Routing design is based on EXOR (extremely opportunistic routing). EXOR is the first program for opportunistic routing, which is an opportunity routing algorithm based on end-to-end shortest path ETX value ${ }^{[7]}$. The basic idea is that the source node wants to send data to the destination node, which first selects the shortest ETX to the destination node which is smaller than its own node as an alternative forwarding node to form an alternative forwarding node set, and then set the priority according to its distance to the destination node, the closer the destination node is, the higher the priority is. The packet carries the alternate forwarding Node IDs which are arranged in the order of priority. The source node broadcasts the packets in batches, and the 
neighbour nodes that received the packets forward the data in the order of priority. If the candidate node with the highest priority is forwarded, the low priority node will not forward the packets again. Instead, it will send the local storage and the higher priority node has not succeeded Send the packet. Each candidate forwarding node forwards this way until the destination node receives most (e.g. 90\%) packets, and the remaining packets are forwarded according to the traditional shortest route.

Since EXOR is the whole network broadcast, the candidate node set is large, the system carries on the stratification to the deployed node set, the EXOR carries on the node candidate only in this layer. In addition, the residual energy is used as another index other than the distance, and if the remaining energy is smaller than the threshold value of the forwarding data, the forwarding path is changed. EXOR energy model is shown in Figure 3.

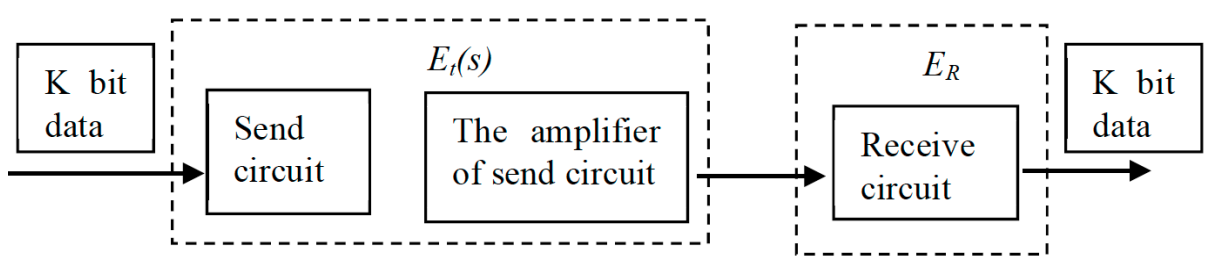

Fig. 3. EXOR energy mode

In this energy model, when the node passes the path of distance $\mathrm{s}$, the energy to be consumed by sending $\mathrm{k}$ data can be calculated by:

$$
\begin{gathered}
E(k, s)=E_{t}(k, s)+E_{R}(k) \\
E_{t}(k, s)=E_{T-\text { elec }}(k, s)+E_{T-\text { amp }}(k, s) \\
E_{R}(k)=E_{R-\text { elec }}(k)=k E_{R-\text { elec }}
\end{gathered}
$$

Based on the residual energy, an energy-based forwarding threshold is given. Where the weight of the energy variable PE is an adjustable value of $0-1$.

$$
M V=\frac{\overline{E T X}}{E T X}(1-P E)+\frac{R E}{M E} P E
$$

Where ETX is the number of packets for the node, and RE is the residual energy, and $\mathrm{ME}$ is the maximum energy of the node.

The simulation experiment is carried out with 10 forwarding nodes as an example, and the obtained path is shown in Table 1. 
Table 1. Forwarding path list

\begin{tabular}{|c|c|c|c|c|}
\hline Node & Reachable node & PE & MV(Threshold) & Forwarding path \\
\hline $\mathrm{S}$ & $1,2,4$ & $0.5,0.4,0.7$ & $0.2,0.3,0.1$ & 2 \\
\hline 2 & $3,5,4$ & $0.2,0.6,0.4$ & $0.1,0.2,0.1$ & 3 \\
\hline 3 & 5,7 & $0.3,0.3$ & $0.4,0.2$ & 7 \\
\hline 7 & 8,9 & $0.4,0.5$ & $0.3,0.2$ & 8 \\
\hline 8 & $9, \mathrm{D}, 6$ & Do not judge & Do not judge & $\mathrm{D}$ \\
\hline
\end{tabular}

$S$ in the table represents a source node, $D$ represents a target node, and a number represents a forwarder node. According to the above route routing algorithm, the forwarding path is $S-2-3-7-8-D$. The application of opportunistic routing with energy threshold has practical significance for the number of routing nodes and the reduction of energy consumption.

\section{Conclusions}

In the software design, the data collection algorithm of high voltage equipment monitoring system is optimized, and fuzzy comprehensive algorithm and opportunistic routing algorithm are applied. As data is being transferred, data thresholds are added to the program in the sensor acquisition node, reducing the amount of redundant data sent. In the design of the coordinator node software, the fuzzy comprehensive evaluation algorithm is applied to the data fusion, which realizes the preliminary processing of the data and reduces the amount of data sent to the sink node. The role of data processing is to reduce the energy consumption of the node, extending the node's life cycle. In the process of data upload, a routing algorithm based on the shortest distance and residual energy is designed to improve the network throughput and extend the network life cycle.

\section{Acknowledgment}

(1) This work is supported by Hebei Agricultural University Science and Technology Fund(LG201604).

(2)This work is supported by Baoding Science Technology Research and Development Guidance Program (15ZG023).

\section{References}

[1] N Harid, AC Bogias, H Griffiths, S Robson, Haddad. A Wireless System for Monitoring Leakage Current in Electrical Substation Equipment[J]. IEEE Access, 2016, 4: 2965-2975 https://doi.org/10.1109/ACCESS.2016.2577553

[2] Dongsheng Zhao, Dong Dai, Licheng L. Electric field energy harvesting for on-line condition-monitoring device installed on high-voltage transmission tower[J]. Electronics Letters, 2015,51(21):1692-1693 https://doi.org/10.1049/el.2015.1975 
[3] CAGD Silva, ELD Santos, ACK Ferrari. A Study of the Mesh Topology in a ZigBee Network for Home Automation Applications[J]. IEEE Latin America Transactions, 2017,15(5), 935-942 https://doi.org/10.1109/TLA.2017.7910209

[4] J Luo. A ZigBee and Sip-Based Smart Home System Design and Implementation[J]. International Journal of Online Engineerin,2017,13(1),42-60.

[5] Z-Stack - ZigBee <www.ti.com.cn> accessed 20.01.2016

[6] Mingyang Zhang, Mingyu Shen. Research of WSN-based data fusion in water quality monitoring[J]. Computer Engineering and Applications, 2014,23,108-119

[7] Zhang R, Gorce JM, Jaffrès-Runser K. Low bound of energy-latency trade-off of opportunistic routing in multi-hop networks[J]. IEEE Computer Society Press, DOI: 10.1109/ICC.2009.5199148 https://doi.org/10.1109/ICC.2009.5199148

[8] P Cheetham, A Hellany, S Jones. Density monitoring of high-voltage SF6 circuit breakers[J]. IEEE Electrical Insulation Magazine, 2015, 31: 6-13 https://doi.org/10.1109/MEI. 2015.7048132

[9] Yang Dan. Detection method of fire smoke video of illumination indoor [D]. Hua Zhong Science and Technology University.2012

\section{Authors}

Yuchen JIA is a teacher of College of Information Science \& Technology, Agricultural University of Hebei, which is in Baoding 071000, China. She is also a Ph.D. Her research topic is the application of wireless sensor networks and performance improvement strategy. Some achievements have been made in the research of wireless sensor networks.

Jia TAO is a technician of College of Information Science \& Technology, Agricultural University of Hebei, which is in Baoding 071000, China. She has done a lot of work on wireless network data acquisition and image processing.

Limin HUO is a professor of College of Mechanical \& Electrical Engineering, Agricultural University of Hebei, which is in Baoding 071000, China. He is also a $\mathrm{PhD}$ supervisor and corresponding author of this paper. He gave a lot of guidance in algorithm design.

Article submitted 08 January 2018. Final acceptance 05 February 2018. Final version published as submitted by the authors. 